\title{
Upgrades toward high-heat flux, liquid lithium plasma-facing components in the NSTX-U
}

\author{
M.A. Jaworski ${ }^{a, *}$, A. Brooks ${ }^{a}$, R. Kaita ${ }^{a}$, N. Lopes-Cardozo ${ }^{b}$, J. Menard $^{a}$, M. Ono ${ }^{a}$, P. \\ Rindt $^{b}$, K. Tresemer ${ }^{a}$ \\ ${ }^{a}$ Princeton Plasma Physics Laboratory, Princeton, NJ, 08543, USA \\ ${ }^{b}$ TU/Eindhoven, Eindhoven, The Netherlands
}

\begin{abstract}
Liquid metal plasma-facing components (PFCs) provide numerous potential advantages over solid-material components. One critique of the approach is the relatively less developed technologies associated with deploying these components in a fusion plasma-experiment. Exploration of the temperature limits of liquid lithium PFCs in a tokamak divertor and the corresponding consequences on core operation are a high priority informing the possibilities for future liquid lithium PFCs. An all-metal NSTX-U is envisioned to make direct comparison between all high-Z wall operation and liquid lithium PFCs in a single device. By executing the all-metal upgrades incrementally, scientific productivity will be maintained while enabling physics and engineering-science studies to further develop the solid- and liquid-metal components. Six major elements of a flowing liquid-metal divertor system are described and a three-step program for implementing this system is laid out. The upgrade steps involve the first high-Z divertor target upgrade in NSTX-U, pre-filled liquid metal targets and finally, an integrated, flowing liquid metal divertor target. Two example issues are described where the engineering and physics experiments are shown to be closely related in examining the prospects for future liquid metal PFCs.

*Corresponding author email: mjaworsk@pppl.gov
\end{abstract}




\section{Introduction}

Liquid metal plasma-facing components (PFCs) provide the potential to solve several outstanding issues for solid-material PFCs. A liquid surface is immune to thermomechanical fatigue, which has been shown to produce cracking and morphological changes in tungsten surfaces[1]. The liquid material itself is also immune to neutron damage and the separation of plasma-material interaction (PMI) processes from the structural material allows one to consider a larger breadth of materials as a substrate, such as nuclear-grade steels[2]. The ability to replenish material in real time with controlled flows of the liquid metal would eliminate net-reshaping of the PFCs due to plasma erosion, transport and redeposition[3]. The improved resilience to transient loading and the elimination of the impact of erosion on lifetime of components could relax many of the requirements on divertor operation[4] and Edge-Localized Mode (ELM) mitigation/elimination[5] in present and future devices.

Candidate materials for use as a liquid metal PFC are, in order of increasing atomic number, lithium, gallium and tin. The Li-Sn alloy is also considered as a liquid metal and exhibits many interesting properties[6]. Liquid lithium, in particular, is widely studied for the following reasons: (a) ease of handling as lithium is compatible with steel over a wide temperature range[2], (b) low $\mathrm{Z}$ nature has resulted in lower $Z_{\text {eff }}$ of core plasmas[7], (c) the chemical reactivity of lithium to common vacuum system residual gases such as oxygen and water vapor results in reduction of these impurities[8], (d) the chemical reactivity of lithium with hydrogen [9] provides a possible means of fueling control where the inventory of lithium within the machine is also controlled[2], and (e) novel confinement regimes are proposed with the use of very low recycling wall conditions obtained with liquid lithium at low temperatures $\left(T<400^{\circ} \mathrm{C}\right)[10]$. Lithium also has the highest vapor pressure of the metals listed above which

provides an additional erosion mechanism in addition to temperature-enhanced sputtering[11, 12]. The ablation of material at elevated materials is believed responsible for transient vapor shielding during experiments conducted on the QSPA plasma gun device[13]. The exploitation of such high-temperature erosion of liquid lithium PFCs has been proposed to 
produce a continuously vapor-shielded target[2] that would provide momentum and energy losses in a scrape-off layer (SOL) as part of a radiating liquid lithium divertor[14].

The National Spherical Torus Experiment-Upgrade (NSTX-U) has developed a research program that includes a conversion to high- $Z$ components and the eventual testing of flowing, liquid metal modules[15]. The major objective of the research program can be summarized as follows: to establish, experimentally, the difference in operations and performance between a machine dominated by high-Z PFCs or a machine operating with low-Z liquid metal PFCs in a single device. Due to the myriad uncertainties associated with machine variations and the relative paucity of high-power, spherical tori, a single-device experiment seems the cleanest method to establish a clear comparison between these two approaches. The use of a spherical torus is also advantageous due to the high surface power densities available. In this way, the NSTX-U program will critically evaluate both, the conventional high-Z approach as well as the novel liquid lithium approach. In order to realize this over-arching goal, a particular set of upgrades has been begun to develop both the high-Z PFCs as well as the flowing liquid lithium systems. The present work first considers the question of the ultimate temperature limit associated with liquid lithium PFCs. It will then summarize the logic associated with this approach as a means of efficiently upgrading a fusion device for high- $\mathrm{Z}$ and liquid lithium experiments. In the course of imagining the upgrade path, certain key experiments will be performed that will inform on the design of liquid lithium PFCs in the future and these will be discussed.

\section{The temperature limit of liquid lithium PFCs}

A key parameter associated with the operation of a liquid metal PFC is the maximum allowable temperature limit. At very high temperatures, temperature-enhanced erosion and evaporation can greatly increase the loss of material from the surface into the nearby plasma[11, 16]. This, however, is not sufficient to indicate liquid metals in such a state are not viable fusion

PFCs. Indeed, it is the goal of numerous research activities to exploit such phenomena to 
improve the performance of the PFC itself.

Figure 1 shows a schematic diagram of the modes of energy transport available to a liquid PFC. Contrasted with solid PFCs, which can only transmit heat by conduction, liquids can utilize both convective transport and phase-change processes to redistribute plasma heat flux. In addition to making use of new channels, many liquid metal concepts attempt to utilize multiple energy transport channels. For example, the proposed, actively-supplied, capillary-restrained scheme would utilize both phase-change (including radiation from the resulting lithium vapor-cloud) as well as conduction cooling to a conventional coolant such as helium or some other fluid[2]. Realizing large densities of lithium in the plasma from evaporation or other processes that intentionally inject lithium may require some form of trapping or differential pumping. One implementation of such a scheme has been called a "vapor-box" system[17]. This scheme would make use of dissipative processes to redistribute plasma heat-flux more uniformly within the divertor region. Heat is then exhausted to a conventional coolant. Surface lithium temperatures over $900^{\circ} \mathrm{C}$ may be required to realize the full potential of the concept.

Similar surface temperatures can significantly increase the effectiveness of liquid metal concepts utilizing fast-flow to convect heat away from the strike-point region. Consider a stream of liquid moving under a uniform heat flux as an approximation of a divertor strike-point impinging a fast-flowing liquid metal. If one considers the liquid metal flow as largely laminar due to the suppression of turbulence by MHD effects[18], then one model for the system is that of a moving slab of material (cavaets of this approach will be discussed below). If the thickness of the fluid flow is larger than the thermal penetration depth, then the temperature rise can be approximated as for a semi-infinite solid:

$$
\Delta T(t)=2 \frac{q_{0} \sqrt{\alpha t}}{k \sqrt{\pi}}
$$

where $\Delta T(t)$ is the surface temperature rise as a function of time, $q_{0}$ is the incident heat flux, $\alpha$ is the thermal diffusivity of the material, and $k$ is the thermal conductivity. The 


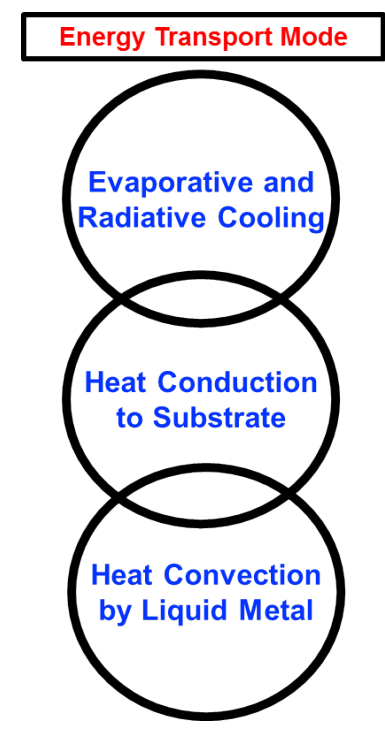

Figure 1: Schematic representation of the modes of energy transport available to liquid metal PFCs. Solid PFCs transfer energy largely via conduction to a coolant. Liquid metal PFCs can transfer energy by phase-change, conduction as well as convective flow and can utilize multiple channels at once.

time of exposure, $\tau_{\text {transit }}$ can be related to a characteristic length-scale in the divertor, $L_{\text {char }}$ and the velocity of the liquid metal, $v_{L M}$ through that region as $\tau_{\text {transit }}=L_{\text {char }} / v_{L M}$. The allowable temperature rise, $\Delta T_{L I M}$ is a function of the limiting temperature for operation in the fusion device and the injection temperature. Rearranging terms, one can solve for the exhaust capacity of the flowing liquid:

$$
q_{0}=\frac{\Delta T_{L I M} k \sqrt{\pi v_{L M}}}{2 \sqrt{\alpha L_{\text {char }}}}
$$

Figure 2 evaluates eq. 2 as pertains to a fast-flowing liquid lithium stream for different values of a limiting surface temperature from $400-900^{\circ} \mathrm{C}$. As can be observed, a higher limiting surface temperature can either relax the requirements on flow velocity or increase the heatexhaust capacity.

While simple, the model does not include several effects that might be important in future implementations, or when attempting to determine a surface temperature rise to a greater degree of accuracy. A constant input heat flux for the period of time $\tau_{\text {transit }}$ in the divertor 


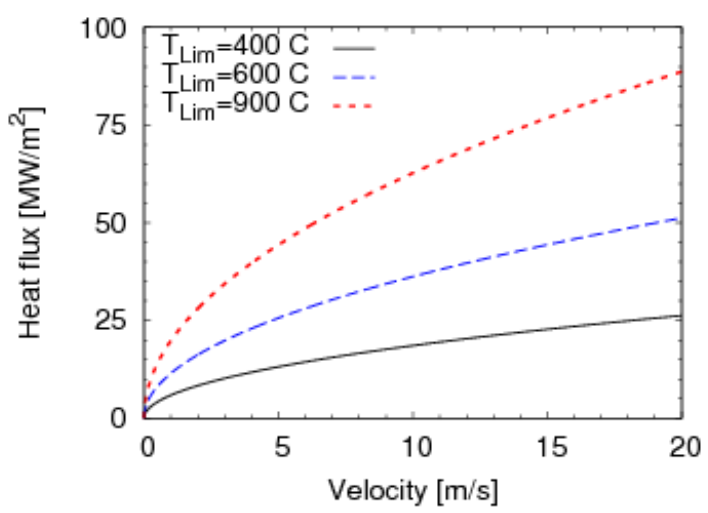

Figure 2: Heat flux exhaust capacity of a fast-flowing liquid lithium stream as as function of stream velocity and limiting surface temperature. The initial temperature is assumed to be $190 \mathrm{C}$ and the characteristic divertor length scale is $10 \mathrm{~cm}$.

length-scale of $L_{\text {char }}$ essentially models the divertor heat flux as a constant step-function near the strike-point. In fact, divertor strike points exhibit complex profiles that arise as a result of transport processes in the plasma[19]. Accounting for this could be accomplished with a time-dependent source term in the governing energy-transport equation, however in this instance the similarity solution used to derive eq. 1 is no longer feasible and other methods must be used (e.g. Fourier transform methods or numerical simulation). The simplistic model used, however, captures the gross effects and conveys the essential purpose and advantage of fast-flow systems as well as the benefits of wider operating temperature windows.

\section{Prospects for high-temperature lithium PFCs}

These analyses highlight the need to identify the ultimate temperature limit of a liquid lithium surface to know what will be compatible with a given plasma scenario (e.g. H-mode) or configuration (e.g. diverted). Understanding the compatibility of this novel regime will depend on addressing the fundamental processes governing material transport in the SOL. A brief overview of available literature will be presented as well as experimental results from studies conducted on the Magnum-PSI linear plasma device[20].

The strong temperature-dependent erosion yields from liquid lithium surfaces results in 
many investigators generating maximum temperature limits for this material. Rognlien and Rensink assumed isothermal wall and divertor conditions with 2-D modeling with the UEDGE code and obtained a temperature limit of $370^{\circ} \mathrm{C}[21]$. Arguments based on a $1 \%$ limit on impurity influx limit create a temperature limit for liquid lithium of approximately $400^{\circ} \mathrm{C}$ though this particle balance criteria is also affected by local redeposition which can extend the allowed temperature range[22]. Both of these works utilize Langmuir law models to describe the evaporation rate of the lithium into the nearby plasma. Experimental work with lithium limiter targets have demonstrated operation of the limiter above $550^{\circ} \mathrm{C}$ before disruption on the FTU tokamak[23]. Consideration of momentum balance in a tokamak SOL yields different results than particle flux estimates. In the case of experiments in the NSTX with measured target plasma electron pressures of 100-200 Pa, the corresponding equilibrium vapor pressure of lithium is reached at approximately $750^{\circ} \mathrm{C}[2]$. Reactor pressures would be expected to reach above $6 \mathrm{kPa}$ which suggests surface temperatures above $900^{\circ} \mathrm{C}[17]$. This wide temperature uncertainty requires experimental verification and would have significant implications on the maximum heat-flux limits of liquid lithium PFCs as well as the efficiency of thermal power cycles interacting with those PFCs - hence this is a key parameter to be examined by the NSTX-U research program. Already there are indications that previous estimates require revision as evidenced by experiments re-examining the lithium erosion yield in high-flux deuterium plasmas[24] and the resulting changes in deuterium transport at high lithium-deuteride concentrations[25]. Two effects are observed in these recent experiments: first, the gross erosion rate is found to be reduced below experiments conducted in low-flux devices and second, a high redeposition rate was calculated indicating strong trapping at the surface[26].

\section{The upgrade path to flowing liquid metal modules}

The NSTX-U is the newest large-scale fusion experiment to come online in the US[27]. The major elements of the upgrade include a second neutral beam heating system and an improved 
Table 1: Multi-machine comparison of size and various powerexhaust metrics. ST-Pilot and ST-DEMO pulse lengths given in terms of discharge-seconds per year.

\begin{tabular}{cccccc}
\hline & $R_{0}$ & $P_{A U X}$ & $P / R$ & $P / S$ & $\tau_{\text {pulse }}$ \\
Machine & $\mathrm{m}$ & $\mathrm{MW}$ & $\mathrm{MW} / \mathrm{m}$ & $\mathrm{MW} / \mathrm{m}^{2}$ & $\mathrm{~s}$ \\
\hline NSTX $^{*}$ & 0.86 & 6.8 & 8 & 0.2 & 1 \\
NSTX-U $^{*}$ & $\mathbf{0 . 9 3}$ & $\mathbf{1 9}$ & $\mathbf{2 1}$ & $\mathbf{0 . 6}$ & $\mathbf{5}$ \\
JET $^{\dagger}$ & 2.95 & 35 & 12 & 0.2 & 20 \\
DIII-D $^{\dagger}$ & 1.74 & 20 & 11 & 0.4 & 6 \\
AUG $^{\dagger}$ & 1.65 & 27 & 16 & 0.6 & 10 \\
CMOD $^{\dagger}$ & 0.7 & 6 & 9 & 0.7 & 2 \\
MAST $^{\dagger}$ & 0.87 & 7.5 & 9 & 0.25 & 1 \\
ITER $^{\dagger}$ & 6.2 & 100 & 16 & 0.15 & 400 \\
ST-Pilot $^{\ddagger}$ & $\mathbf{2 . 2}$ & $\mathbf{1 9 0}$ & $\mathbf{8 6}$ & $\mathbf{0 . 7}$ & $6 \times 10^{6}$ \\
ST-DEMO $^{\ddagger}$ & $\mathbf{3 . 2}$ & $\mathbf{5 2 0}$ & $\mathbf{1 6 1}$ & $\mathbf{0 . 9}$ & $\infty$ \\
\hline
\end{tabular}

* J.E. Menard, et al., Nucl. Fusion 52 (2012) 083015.

$\dagger$ Th. Eich, et al., "Scaling of the tokamak near scrape-off layer H-mode power width and implications for ITER", ITPADiv/SOL group ITR 1/1, San Diego, October, 2012.

$\ddagger$ J.E. Menard, et al., Nucl. Fusion 51 (2011) 103014.

center-stack. The center-stack upgrade enables toroidal fields of 1 T, plasma currents of 2 MA and pulse-lengths of $5 \mathrm{~s}$. The second neutral beam heating system effectively doubles the available input power resulting in a maximum available heating capacity of $19 \mathrm{MW}$. These upgrades place NSTX-U in a unique position relative to other fusion devices world-wide. Two metrics of interest are the $P / R$ and $P / S$ parameters of each machine meaning the available input power, $P$, divided by the machine major radius, $R$, or the machine surface area, $S$. Table 4 provides a comparison of NSTX-U with a number of machines current or planned. The compact, high- $\beta$ nature of the NSTX-U makes it possible to reach very high values of both metrics.

Another feature of the NSTX-U is the open floor divertor configuration. This divertor, while sacrificing the dissipative benefits of a closed divertor, makes it possible to explore a wide variety of different plasma shapes and PFC options. Already, the NSTX had made use of this flexibility in testing the Liquid Lithium Divertor (LLD)[28, 29]. This high-Z PFC surface was placed in an outboard location such that high-triangularity, high-performance 


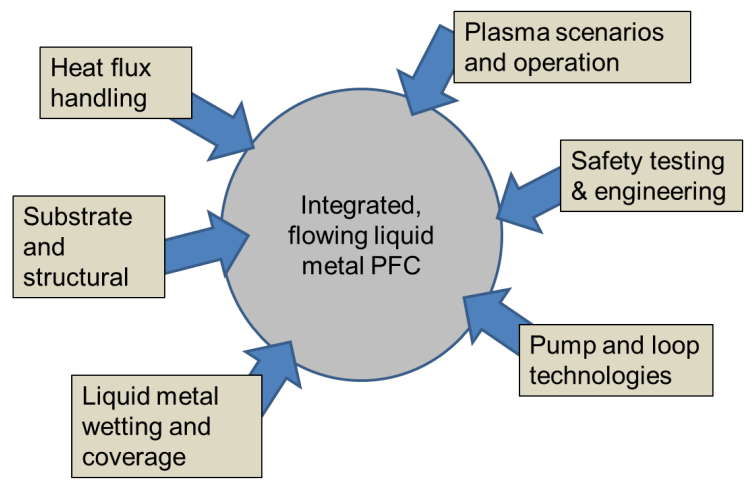

Figure 3: Six major elements are described as part of the necessary steps in developing liquid metal plasma-facing components for the NSTX-U. See text for details.

discharges would place the outboard strike-point at a considerable distance $(\simeq 30 \mathrm{~cm})$ from the LLD. Later, for specific experiments on the LLD, the magnetic shaping flexibility of NSTX enabled experiments with the strike-point directly impinging. This flexibility in the experimental facility makes it possible to accommodate such significant PFC upgrades as the LLD while continuing to enable a significant portion of the research program on core physics studies.

A similar logic as was used with the LLD is proposed for upgrading the NSTX-U to high-Z PFCs. In 2015, the NSTX-U will begin operations with all graphite surfaces. PFC upgrades will initially be placed at an outboard location to preserve experimental capabilities and avoid limits on operations for high-triangularity discharges. Over the course of approximately five years, the machine would be converted to all, high-Z PFCs beginning with a portion of the outboard divertor, to complete conversion of the outer half of the lower vessel, extending then to include the outer and inner first-wall PFCs, and then finally the remaining lower and upper divertor areas will be converted. This plan retains graphite in the highest heat flux areas for a maximum amount of time due to its robust performance as a PFC.

Just as the upgrades to the in-vessel PFCs is staged, implementation of a flowing liquid lithium module would be difficult to realize in a single step. Instead, portions of the technology are implemented in steps to confirm engineering design and analysis of the PFCs. The 
major elements required for a confinement device, liquid metal PFC are shown in figure 3. Six major elements are expected to be required in creating an integrated module with flowing liquid metal for the divertor of a confinement device. Beginning in the top left of the figure and proceeding counter-clockwise these are described as follows:

1. the PFC structure itself must be capable of absorbing, or otherwise intercepting a significant (i.e. divertor-relevant) heat flux and dissipating it or otherwise avoiding permanent damage,

2. the PFC substrate and structure itself must withstand thermomechanical and electromagnetic loads, the PFC substrate material itself must be chemically compatible with the liquid metal in the expected temperatures of use,

3. the liquid metal must adhere to the surface of the substrate in order to be wetted and maintain coverage of the PFC itself,

4. technologies necessary to moving the liquid metal throughout a system and into and out of vacuum must be developed and demonstrated in order to be integrated into a flowing system,

5. safety systems and procedures associated with the liquid metal handling technologies as well as in-vessel components must be established, and

6. plasma scenarios and operational control needs to be developed to account for the changes that may arise with the use of the liquid metal PFC including the elimination or acceptability of ELMs and other transients.

While significant development on most of these items can be accomplished in a laboratory environment, item \#6 explicitly requires a confinement device. Development of reactorrelevant PFCs would additionally require attention to active-cooling schemes compatible with the substrate loading and impact integration of the PFCs into the confinement device, though this is not required for NSTX-U. In addition, the operation of a confinement device with a 
high-vapor pressure divertor target is still speculative at present. Other liquid metal concepts would be required if experimental tests indicated it would not be possible to operate at hightemperatures with good core performance. In the extreme, if high-temperature lithium could not be used and divertor heat-fluxes could not be reduced through other means, then a fastflowing system would be required for Li or an alternative metal such as Sn would need to be used. These fast-flowing system would be a significantly different approach, technically, than slowly-flowing or oozing systems.

Balancing all these needs, the approach currently favored for the NSTX-U program is a set of incremental upgrades that will, in turn, establish the feasibility of new operating scenarios while gradually increasing the complexity of the liquid metal system. These upgrades involve the following:

1. a continuous row of high-Z PFC tiles in the NSTX-U divertor,

2. the implementation of pre-filled, liquid-metal PFC target tiles in the same region, and

3. a fully-integrated, flowing liquid metal system

Each of these upgrades will address certain key technological and scientific questions described next.

\subsection{High-Z divertor target upgrade}

The first major upgrade is the high-Z PFC tile upgrade for the NSTX-U divertor. The current PFCs are composed of an amorphous graphite (type "ATJ" manufactured by Union Carbide). Upon application of a lithium layer, the lithium rapidly diffuses (or "intercalates") into the graphite resulting in a loss mechanism not related to plasma erosion or evaporation. While the diffusive process is temperature dependent, lithium penetration into the graphite substrate was observed to occur rapidly, on the order of minutes, even at room temperature[30]. Experiments comparing lithium coating lifetimes on graphite vs. a molybdenum alloy (TZM) substrate found a factor of 100 change in the available lithium at the 
surface of the substrate[26]. This indicates that the metallic substrate will serve as a more substantial source of lithium for plasma experiments than would be expected from graphitic surfaces. In addition, the high-Z tiles will provide a compatible substrate that can resist the thermomechanical and electromagnetic forces it will be subjected to during plasma bombardment at divertor-relevant heat fluxes. The high-Z divertor tile surfaces are expected to reach over $1200^{\circ} \mathrm{C}$ during experiments placing the strike-point directly on the high-Z tiles - cooling is by thermal inertia. The high-Z tile design and analysis are detailed elsewhere[31].

Lithium will continue to be applied by evaporation from the NSTX LITER systems[32]. Experiments conducted in the Magnum-PSI on evaporated coatings on TZM molybdenum found erosion rates under high-flux deuterium bombardment significantly below erosion from pure Li[24]. The first experiments in NSTX-U will provide a confinement-device analogue to these experiments to confirm whether the gross erosion is suppressed. Very high redeposition rate, $R>0.99$, indicating a strong trapping effect at the PFC surface. Repetition of these conditions in the tokamak will compare the normal-incidence magnetic field experiments in Magnum-PSI with oblique-incidence magnetic field conditions found in a tokamak divertor. These experiments at high-temperatures will begin to address the overall plasmacompatibility (e.g. confinement properties) with these limited-inventory, lithium coatings on high-Z substrates.

\subsection{Pre-filled liquid metal divertor targets}

The second major upgrade and experiment will involve a novel PFC system: a pre-filled liquid metal target. In concept, these devices are similar to Capillary-Porous Systems developed in the Russian Federation[33] and deployed on several devices[7]. A schematic of such a PFC target is shown in figure 4. The key difference between this present concept and that of a CPS is that we are targeting a divertor-relevant PFC whereas CPS devices are typically inserted as movable limiter probes. The KTM is a proposed device under construction to examine a divertor target utilizing CPS-like technologies but has yet to operate[34]. Schematically, 


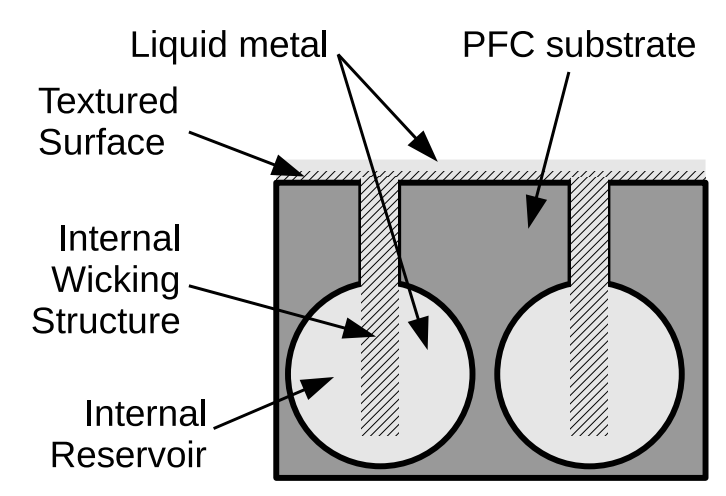

Figure 4: Schematic representation of the pre-filled, liquid metal divertor target. The PFC substrate would be modified to include internal reservoir and wicking structures so that liquid metal could be supplied to the free surface. Wetting and spreading is enhanced by surface texturing. Realization as an actual divertor target would require addition of mounting features not included in this schematic.

then, the PFC would include an internal liquid metal reservoir and a wicking structure to allow transport of material from the internal spaces to the free-surface. Wetting and spreading on the surface is facilitated by surface texturing. Heat flux is absorbed by the target's thermal inertia, as was utilized in the high-Z divertor upgrade. Similar temperatures as in the previous upgrade should be achievable with the pre-filled targets. Technologically, this upgrade will achieve the introduction of lithium into the NSTX-U without the use of the LITER evaporation system and realize the production of a complex, liquid metal PFC divertor target.

The scientific goals of the prefilled targets are to establish the viability of passive-replenishment strategies for the slowly-flowing concepts. This includes demonstration of proper wetting of the liquid metal on the substrate, replenishment from sub-surface reservoirs and mitigation of the effects of contamination in the liquid metal. It will also further establish the compatibility of high-temperature lithium targets with the plasma. In contrast to the experiments on the high-Z tiles previously described, the prefilled targets will each have reservoir capacities in the several grams of lithium range whereas the in-vessel deposition with the LITER system is on the order of 100-500 milligrams prior to each plasma discharge in NSTX-U. In other 
words, the limited inventory of evaporated coatings may provide an optimistic assessment of high-temperature lithium compatibility whereas a pre-filled target will more closely mimic a fully-flowing system in available lithium inventory.

\subsection{Fully-integrated, flowing divertor targets}

As a final upgrade, liquid metal handling technologies would be integrated to the confinement device PFCs creating a fully-flowing, liquid metal PFC. The technical progress with respect to the PFC itself is modest if internal cooling is not included as already the pre-filled target schematic looks similar to actively-cooled concepts shown in ref. [2]. In the context of the NSTX-U where only $5 \mathrm{~s}$ discharges are planned in the near-term, inertial cooling should be sufficient for experiments as utilized for the high-Z PFCs described above in sect. 4.1. In particular, temperatures as high as $1200^{\circ} \mathrm{C}$ will be available based on initial calculations[31] for the current design of high-Z tiles, but the pre-filled target experimental campaign is expected to determine what temperatures are compatible with good core performance. For an oozing-flow system, techniques will have to be developed to re-collect excess fluid that has passed over the PFC or deposited onto other surfaces. Significant technical effort is required, however, to integrate an external liquid metal loop system to the PFCs and circulate the liquid.

Scientific progress is also modest in that one would expect most of the questions of plasma compatibility to have been addressed with the pre-filled targets. However, integration with a liquid-metal pumping system provides the ability to enhance the passive replenishment of liquid metal with active means (i.e. pressurization). An important goal for this stage of the project would be an assessment of the liquid metal inventory, transport and control during normal and off-normal plasma operations. Such inventory control would be essential for future reactor concepts[2].

The benefit, therefore, of the pre-filled target program is to separate technical risks associated with the fully-flowing liquid targets. The technical implementation of the pre-filled 
targets already addresses issues associated with the plasma-material interface and behavior of the PFCs in the tokamak. This allows technical efforts for the fully-flowing system to focus on the external loop system and integration while useful scientific studies are carried out in the tokamak.

\section{Experimental interpretation and design of components}

Two example research problems detailing particular questions facing the high-Z divertor and pre-filled target experiments are included. These samples are included in that they pertain specifically to a high-temperature development program and one where sacrificial layers are emphasized. Additional research and experiments will further explore issues related

to interpretation and conduct of experiments. In particular, these two elements highlight some of the difficulties in engineering design of these components and how they impact the scientific program and vice versa. Other aspects will not be covered. For instance, it is known that with dominant carbon surfaces, a signficant carbon fraction is still expected in the NSTX-U plasmas, of order 1-3\%. These impurities will influence material erosion rates from the PFCs and have an impact on physics results. However, this is part of the experimental campaign and does not directly influence the design of components and is not further discussed in this work.

\subsection{Leading edge effects on lithium experiments}

An inhomogenous divertor target will produce leading edges in the absence of special geometries such as fish-scaling. The NSTX-U divertor is composed of divertor tile targets that do not have fish-scaling geometries in order to preserve the capability of operating with the toroidal magnetic field in the opposite direction. As a result, some attention is necessary to the production and mitigation of leading-edge effects.

A schematic view of the geometry involved at a gap between divertor PFCs is shown in figure 5. The magnetic field line is incident on the nominal PFC surface at an angle $\alpha$ to the 


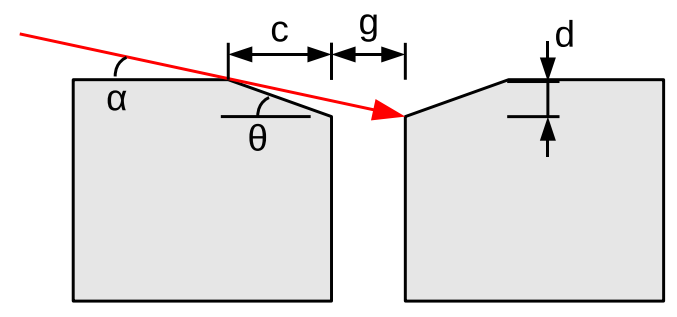

Figure 5: Schematic diagram of a chamfer for mitigating leading edges.

surface. As expected, for a nominal parallel heat flux, $q_{\|}$, the surface heat-flux, $q_{0}$ is related to $q_{\|}$by the total angle of incidence as

$$
q_{0}=q_{\|} \sin (\alpha)
$$

Typical divertor angles of incidence are in the range of $1-5^{\circ}$. At a sharp corner, then, the angle changes to $\alpha+90^{\circ}$ resulting in $q_{0} \simeq q_{\|}$. This region of enhanced heat flux is limited in area, but can lead to significant heating of the leading edge, even for relatively small values of gap space, $g$.

One mitigation strategy is to eliminate the leading edge by introducing a chamfered edge as shown in figure 5. This chamfer geometry eliminates the leading edge at the cost of an increased total angle, $\alpha+\theta$ on the following chamfer face. For small angles, the enhancement in heatflux over the nominal front-face heat flux, $f_{\text {enh }}$ is given as follows:

$$
f_{\text {enh }}=\frac{q_{\|} \sin (\alpha+\theta)}{q_{\|} \sin (\alpha)} \simeq \frac{\alpha+\theta}{\alpha}
$$

In the case that a single angle of incidence is known and the gap, $g$ is known, then the 
chamfer width, $c$, and depth, $d$ can be calculated to exactly shadow the next leading edge:

$$
\begin{aligned}
c & =g \frac{\tan (\alpha)}{\tan (\theta)-\tan (\alpha)} \\
d & =c \tan (\theta)
\end{aligned}
$$

In practice, however, manufacturing tolerances will add to both the gap size as well as introduce a potential vertical displacement. In addition, a range of values for $\alpha$ can be imagined as a result of different tokamak geometries during experiments. In the course of the high-Z design project, both manufacturing tolerances and installation tolerances have been accounted for and impact the final choice for chamfer geometry. The most critical feature is the vertical misalignment between tiles as a horizontal misalignment is reduced by the sine of the incident field line, $\alpha$.

A theoretical minimum exists in which the trailing edge chamfer angle exactly equals in the nominal angle of incidence leading to $f_{e n h} \geq 2$. In practice, accounting for finite tolerances, values of $f_{e n h} \simeq 2.3$ are typical. From eq. 5 one can see that as the chamfer angle, $\theta$ approaches the value of $\alpha$ the width of the chamfer, $c$, grows large - another practical issue that limits the value of $f_{\text {enh }}$. Values of less than two can be achieved in alternate geometries such as a ramped scheme variation but this does not support bi-directional operation.

While these relationships are fairly standard in the field, there is a particular impact the leading-edge heating effects have in the context of lithium experiments. The temperature rise on the PFC surface scales as the incident heat flux (c.f. eq. 1). The surface average temperature, $\langle T\rangle$, including increases due to the presence of chamfer features can be calculated as follows:

$$
<T>=\frac{A_{\text {nom }} T_{\text {nom }}}{A_{\text {div }}}+\frac{A_{\text {ch }} T_{\text {enh }}}{A_{\text {div }}}=\left(1-f_{\text {ch }}\right) T_{\text {nom }}+f_{c h} T_{\text {enh }}
$$

where $A_{\text {nom }}, A_{c h}$, and $A_{d i v}$ are the total nominal (flat) surface area, the total chamfer surface area and the total divertor surface area respectively. The fraction of surface area at an 


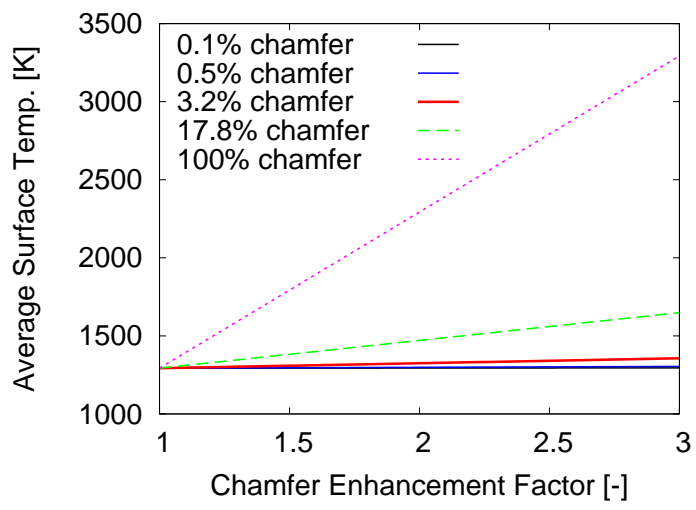

Figure 6: The surface average temperature as a function of chamfer enhancement factor at different chamfer fractional areas.

enhanced temperature is $f_{c h}=A_{c h} / A_{\text {div }}$. The nominal surface temperature is $T_{\text {nom }}$ whereas the temperature on the chamfer surfaces is $T_{e n h}$. As it is the temperature rise that scales with incident heat flux, the enhanced temperature due to a given heat flux enhancement factor is given as:

$$
T_{\text {enh }}=T_{0}+f_{\text {enh }} \Delta T_{\text {nom }}
$$

whereas $T_{\text {nom }}=T_{0}+\Delta T_{n o m}$ with $T_{0}$ being the initial temperature. Figure 6 shows the relationship between the surface average temperature and the heat-flux enhancement factor for a range of values of $f_{c h}$.

While the surface average temperature is a completely linear relationship, there is an additional effect to consider when conducting lithium experiments. In the case of a surface covered in a liquid, the evaporative flux, $J(T)$ is calculated from as follows[35]:

$$
J(T)=\frac{P_{v}(T)}{\sqrt{2 \pi m k T}}
$$

where $P_{v}(T)$ is the equilibrium vapor pressure at temperature $T, m$ is the atomic mass of the liquid, and $k$ is the Boltzmann constant. The vapor pressure of lithium in Pascal is given $\operatorname{as}[36]$ :

$$
P_{v}(T)=133.3 \exp \left(18.4-\frac{18750}{T}\right)
$$




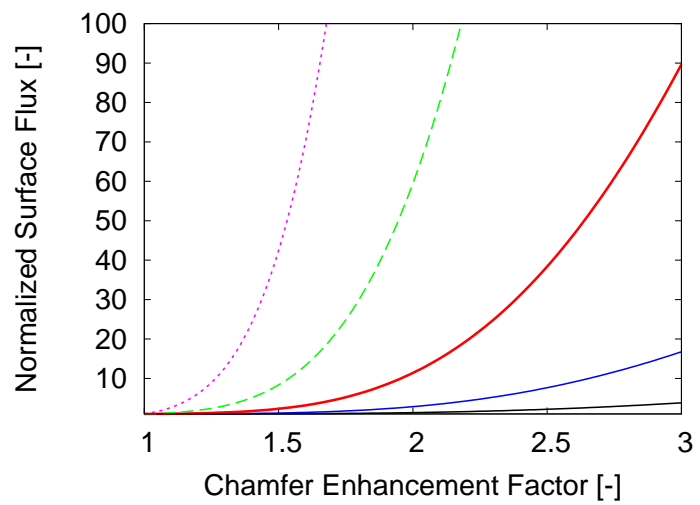

Figure 7: The surface average temperature as a function of chamfer enhancement factor at different chamfer fractional areas. Lines symbols correspond to those in fig. 6 .

where $T$ is expressed in Kelvin.

The surface flux of material, $\langle J\rangle$, due to evaporation can be constructed as follows:

$$
<J>=J\left(T_{\text {nom }}\right)\left(1-f_{c h}\right)+J\left(T_{c h}\right) f_{c h}
$$

Figure 7 shows the result of this calculation normalizing the average particle flux to $J\left(T_{\text {nom }}\right)$. The effect of the exponential response in vapor pressure is clearly visible in the figure. For instance, a PFC geometry with only $3.2 \%$ chamfer surface area and $f_{\text {enh }}=2.5$ will experience a 40-fold increase in particle flux from the surface due to the local temperature peaking. As a result, experiments conducted on PFCs exhibiting leading-edge heating will require some careful interpretation as not only will the surface fluxes be larger than expected, these conditions may also be transient as some portions of the PFC-face exhibit an increased material loss from some regions and not others leading to inhomogeneous depletion.

\subsection{Porous-MHD effects and surface replenishment}

Both the CPS system and the divertor targets proposed by the NSTX-U group feature liquid metal flow through a porous material. The porous material is advantageous for fluid stability[29] and can provide a passive means of replenishing a surface. This is the phe- 
nomenon of porous material imbibition[37]. Flow through a porous material can be described by the Darcy equation:

$$
u=\frac{k \Delta P}{\mu L}
$$

where $u$ is the fluid velocity, $k$ is the permeability of the porous material, $\Delta P$ is a pressure differential, $\mu$ is the fluid viscosity and $L$ is some distance over which $\Delta P$ is present. In the case of a simple porous material and wetting liquid ${ }^{1}$ the pressure at the interface between the non-wetted and wetted portions of the porous material are given as the capillary pressure, $P_{C}:$

$$
P_{C}=\frac{2 \Sigma \cos (\gamma)}{r_{p}}
$$

where $\Sigma$ is the surface, or interfacial, tension of the liquid, $\gamma$ is the contact angle of the liquid on the porous substrate and $r_{p}$ is the mean pore radius. In the case of a moving fluid of electrical conductivity, $\sigma$, in an applied magnetic field, $B$, an additional pressure drop over the length of moving fluid is approximated as:

$$
P_{M H D} \simeq-\sigma u B^{2} L
$$

An MHD version of the Lucas-Washburn equation is formed by including both capillary and MHD pressure terms in the Darcy equation and rearranging as:

$$
L u=L \frac{\mathrm{d} L}{\mathrm{~d} t}=\frac{2 \Sigma \cos (\gamma)}{r_{p}} \cdot \frac{1}{\mu / k+\sigma B^{2}}
$$

Including a definition of the Hartmann number using the average pore radius as the characteristic length, $\mathrm{Ha}=r_{p} B \sqrt{\sigma / \mu}$, one can solve for the location of the wicking front as a function of time:

$$
L(t)=\left(\frac{4 \Sigma r_{p} \cos (\gamma)}{\mu\left(r_{p}^{2} / k+\mathrm{Ha}^{2}\right)} t\right)^{1 / 2}=S \sqrt{t}
$$

where $S$ is known as the sorptivity of the porous-MHD system.

\footnotetext{
${ }^{1}$ A "wetting liquid" is here defined as one for which the contact angle is less than $90^{\circ}$.
} 
We provide here a small note on the relevance of capillary pressure to this analysis and the role of additional pressure heads. In the case of a passive system, such as the pre-filled divertor target concept, the only filling force available is capillary pressure. For future implementations of fully-flowing systems, some positive pressure head could be applied to force fluid through the porous material. Equation 15 is applied even in a fully wetted system for the simple reason that a wetted system experience losses from a surface will not remain "fully wetted" for all time without some replenishment mechanism. In fact, the literature on porous flowing materials indicates that the capillary pressure associated with porous materials is a function of the saturation of those pores and increases as material is removed[37]. Additional terms have been considered, in principle, and are reported in the literature as well[13]. In the case of a passively filled system, though, the estimated capillary pressure of a $100 \mu \mathrm{m}$ pore filled with liquid lithium at $250^{\circ} \mathrm{C}$ is about $6.4 \mathrm{kPa}$. This is approximately 64 times larger than the previously measured plasma pressure of the NSTX divertor many orders of magnitude greater than the neutral pressure in that device[2]. It is appropriate, therefore, to consider only capillary pressure and MHD damping terms.

While instructive, more insight into the importance of the results associated with eq. 16 can be obtained. Returning to eq. 15, one can combine terms to create a modified definition of the Hartmann number taking into account the effectively enhanced viscosity due to the permeability of the porous material:

$$
\chi=\frac{k}{r_{p}^{2}} \mathrm{Ha}^{2}
$$

where $\chi$ is the permeability-enhanced Hartmann number. Using this definition, eq. 15 is rearranged to yield:

$$
L \frac{\mathrm{d} L}{\mathrm{~d} t}=\left(\frac{2 \Sigma \cos (\gamma)}{B \sqrt{\sigma \mu}}\right) \cdot \frac{\sqrt{k}}{r_{p}} \cdot \frac{\sqrt{\chi}}{1+\chi}
$$

This form allows one to optimize a porous material for a given magnetic field and other fluid properties. This is done by maximizing the fluid flow which means finding the maximum of 


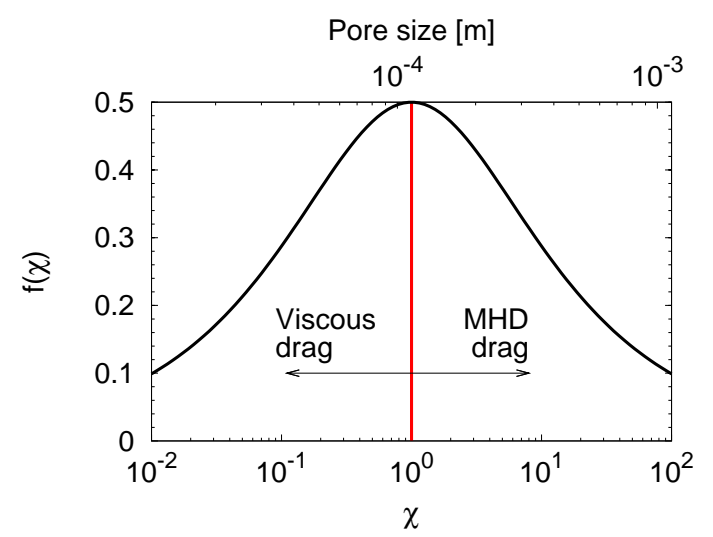

Figure 8: Plot of the function $f(\chi)=\sqrt{\chi} /(1+\chi)$ showing the optimization at a value of $\chi=1$. Flow through systems below this value are dominated by viscous drag whereas flows in systems above this value are dominated by MHD drag. Using the packed spheres approximation for a porous medium and liquid lithium properties in a $1 \mathrm{~T}$ field, a pore-size corresponding to the range of $\chi$ is shown as the second abscissa at the top of the plot.

the function $f(\chi)=\sqrt{\chi} /(1+\chi)$. The maximum of this function is easily found to be $\chi=1$. As with more conventional MHD problems, this optimization indicates that a maximum of flow is found at the point that the viscous drag is equal to the MHD drag for the particular geometry of the system. This is illustrated in figure 8 .

As an illustration, a common approximation for the permeability of a packed-bed of spheres with porosity of $\epsilon=0.5$ is estimated with the Kozeny-Carman equation[37]:

$$
k=\frac{\epsilon^{3}}{45(1-\epsilon)^{2}} r_{p}^{2}
$$

with liquid lithium properties at $250^{\circ} \mathrm{C}$ and a field of $1 \mathrm{~T}$. This would indicate an optimal pore size of about $130 \mu \mathrm{m}$. We note that the pore sizes used in the LLD esd estimated to be about $\approx 20 \mu \mathrm{m}[2]$.

The MHD effects create an anisotropic sorptivity even if the porous material is isotropic throughout. In the directions perpendicular to the magnetic field, the MHD damping reduces the flow rate while parallel to the magnetic field only viscous drag occurs. As a result one can evaluate the sorptivity for the parameters above and find that $S_{\perp}^{2}=16 \mathrm{~cm}^{2} \mathrm{~s}^{-1}$ and $S_{\|}^{2}=38$ 
$\mathrm{cm}^{2} \mathrm{~s}^{-1}$. These values have a practical impact on the design of prefilled or fully flowing PFCs of the variety that have oozing pores communicating material from a reservoir or internal pipe of lithium. Equation 16 can be rearranged such that a characteristic time, $\tau_{\text {replenish }}$ to replenish a surface over the distance between pores, $L_{\text {pore }}$ can be calculated:

$$
\tau_{\text {replenish }}=\frac{L_{\text {pore }}^{2}}{S^{2}}=\frac{1}{f_{\text {replenish }}}
$$

where a replenishing frequency, $f_{\text {replenish }}$ can be taken from the characteristic time. For a characteristic scale length of $1 \mathrm{~cm}$ the replenishment frequency is in the range of $16-38 \mathrm{~Hz}$ depending on parallel or perpendicular flow for the optimal pore size in a $1 \mathrm{~T}$ field. This range of frequencies is already close to the typical ELM frequency in tokamak devices so that if an ELM event were severe enough to completely ablate all the surface lithium, the wicking process would be expected to recover the surface prior to the next ELM event.

While the pore sizes and features of the porous target are small, attention is still required to leading edge issues. The reason for this lies in the discrete nature of divertor targets. For features on the tile target itself, leading edges can often be ignored due to the small scale. However, the structure on which components are mounted is sometimes irregular and can generate gaps of a variable nature. Complex installation procedures can be utilized to alleviate any irregularities, but these can also become costly due to the labor required. It therefore remains prudent to design against leading edges as described in section 5.1

\section{Summary}

The potential benefits of liquid metal, and in particular liquid lithium, plasma-facing components has been re-iterated. The NSTX-U program has a particular focus on making a critical assessment of the conventional high-Z wall approach alongside the low-Z, liquid metal PFC approach in a single device. Of particular importance in establishing the feasibility of liquid lithium as a fusion PFC is determining the operating temperature window. In the area of 
power-exhaust capacity, the maximum temperature compatible with good core confinement could have significant impact on the direction of development for future liquid metal PFCs.

The NSTX-U is well suited to testing liquid metal PFCs due to the large heating power available as well as the flexible magnetic configurations. A three-step upgrade path has been described that will address the key elements required to implement fully-flowing liquid lithium

PFCs in the NSTX-U beginning with the high-Z divertor upgrade. Scientific and technological progress will be made at each upgrade. Two specific issues are given to illustrate some of the research questions associated in these first two steps.

In the NSTX-U high-Z divertor upgrade, leading edges will be mitigated with the use of a chamfer shaping at the tile edges. The geometric construction of these chamfers was described. While the increase in average surface temperature is nearly trivial to evaluate, a significant increase in surface particle flux is expected due to the exponential response of the lithium vapor-pressure to the surface temperature. The pre-filled liquid metal target has been described in concept. A key question for this and other concepts utilizing a porous medium is how to account for the effects of MHD drag inside a porous material. An analysis is presented deriving an MHD-modified Lucas-Washburn equation which results in anisotropic sorptivities. The sorptivity can be maximized, however, to provide the fastest possible wicking through the material. Replenishment rates from reasonably sized pores are commensurate with typical ELM cycle times indicating rapid replenishment of liquid metals is feasible even inter-ELM.

\section{Acknowledgments}

This work is supported under the U.S. Department of Energy contract \#DE-AC02-09CHI1466 and PPPL Laboratory Directed Research and Development (LDRD) funding. Some of us (M.A.J.) would like to also thank R. Maingi and R. Majeski for many stimulating hours developing liquid metal strategy which informed portions of this manuscript. 


\section{References}

[1] M. Wirtz, J. Linke, G. Pintsuk, G. De Temmerman, G. M. Wright, Thermal shock behaviour of tungsten after high flux h-plasma loading, J. Nucl. Mater. 443 (2013) 497510.

[2] M. A. Jaworski, A. Khodak, R. Kaita, Liquid-metal plasma-facing component research on the National Spherical Torus Experiment, Plasma Phys. Control. Fusion 55 (2013) 124040.

[3] P. Stangeby, Assessing material migration through ${ }^{13} \mathrm{C}$ injection experiments, J. Nucl. Mater. 415 (2011) S278-S283.

[4] M. Wischmeier, et al., High density operation for reactor-relevant power exhaust, J. Nucl. Mater. 463 (2015) 22-29.

[5] A. J. Thornton, A. Kirk, P. Cahyna, I. T. Chapman, G. Fishpool, J. R. Harrison, Y. Q. Liu, L. Kripner, M. Peterka, ELM mitigation via rotating resonant magnetic perturbations on MAST, J. Nucl. Mater. 463 (2015) 723-726.

[6] R. Bastasz, J. Whaley, Surface composition of liquid metals and alloys, Fusion Engineering and Design 72 (2004) 111-119.

[7] M. L. Apicella, G. Mazzitelli, V. Pericoli Ridolfini, V. Lazarev, A. Alekseyev, A. Vertkov, R. Zagórski, FTU Team, First experiments with lithium limiter on ftu, Journal of Nuclear Materials 363-365 (2007) 1346-1351.

[8] G. S. Xu, B. N. Wan, J. G. Li, X. Z. Gong, J. S. Hu, J. F. Shan, H. Li, D. K. Mansfield, D. A. Humphreys, V. Naulin, Study on H-mode access at low density with lower hybrid current drive and lithium-wall coatings on the EAST superconducting tokamak, Nucl. Fusion 51 (2011) 072001. 
[9] M. J. Baldwin, R. P. Doerner, S. C. Luckhardt, R. W. Conn, Deuterium retention in liquid lithium, Nuclear Fusion 42 (2002) 1318-1323.

[10] R. Majeski, R. Doerner, T. Gray, R. Kaita, R. Maingi, D. Mansfield, J. Spaleta, V. Soukhanovskii, J. Timberlake, L. Zakharov, Enhanced energy confinement and performance in a low-recycling tokamak, Phys. Rev. Lett. 97 (2006) 075002.

[11] R. P. Doerner, et al., Measurement of erosion mechanisms from solid and liquid materials in PISCES-B, Journal of Nuclear Materials 290-293 (2001) 166-172.

[12] J. P. Allain, M. D. Coventry, D. N. Ruzic, Enhanced energy confinement and performance in a low-recycling tokamak, Phys. Rev. B 76 (2007) 205434.

[13] V. A. Evtikhin, I. E. Lyublinski, A. V. Vertkov, S. V. Mirnov, V. B. Lazarev, N. P. Petrova, S. M. Sotnikov, A. P. Chernobai, B. I. Khripunov, V. B. Petrov, D. Y. Prokhorov, V. M. Korzhavin, Lithium divertor concept and results of supporting experiments, Plasma Physics and Controlled Fusion 44 (2002) 955-977.

[14] M. Ono, M. A. Jaworski, R. Kaita, H. W. Kugel, et al., Recent progress in the NSTX/NSTX-U lithium programme and prospects for reator-relevant liquid-lithium based divertor development, Nucl. Fusion 53 (2013) 113030.

[15] J. E. Menard, et al., NSTX upgrade five year plan for FY2014-FY2018, Tech. rep., Princeton Plasma Physics Laboratory (May 2013).

[16] J. P. Allain, et al., Temperature dependence of liquid-lithium sputtering from oblique $700 \mathrm{eV}$ He ions, J. Nucl. Mater. J. Nucl. Mater. (2003) 641-645.

[17] R. J. Goldston, R. Myers, J. Schwartz, The lithium vapor box divertor, Physica Scripta T167 (2016) 014017. 
[18] N. B. Morley, S. Smolentsev, L. Barleon, I. R. Kirillov, M. Takahashi, Liquid magnetohydrodynamics - recent progress and future directions for fusion, Fusion Eng. Des. $51-52(2000) 701-713$.

[19] T. Eich, et al., Scaling of the tokamak near the scrape-off layer H-mode power width and implications for ITER, Nuclear Fusion 53 (2013) 093031.

[20] G. De Temmerman, M. A. van den Berg, A. Lof, H. J. van der Meiden, H. J. van Eck, T. W. Morgan, T. M. de Kruijf, P. A. Zeijlmans van Emmichoven, J. J. Zielinski, High heat flux capabilities of the Magnum-PSI linear plasma device, Fusion Eng. Des. 88 (2013) 483-487.

[21] T. D. Rognlien, M. E. Rensink, Impurity transport in edge plasmas with application to liquid walls, Phys. Plasmas 9 (2002) 2120-2126.

[22] J. W. Coenen, G. De Temmerman, G. Federici, V. Philipps, G. Sergienko, G. Strohmayer, A. Terra, B. Unterberg, T. Wegener, D. C. van den Bekerom, Liquid metals as alternative solution for the power exhaust of future fusion devices: status and perspective, Phys. Scr. T159 (2014) 014037.

[23] M. Apicella, et al., Lithization of the FTU tokamak with a critical amount of lithium injection, Plasma Phys. Control. Fusion 54 (2012) 035001.

[24] T. Abrams, M. A. Jaworski, M. Chen, E. A. Carter, R. Kaita, D. P. Stotler, G. De Temmerman, T. W. Morgan, M. A. van den Berg, H. J. van der Meiden, Suppressed gross erosion of high-temperature lithium via rapid deuterium implantation, Nucl. Fusion 56 (2016) 016022.

[25] M. Chen, T. Abrams, M. A. Jaworski, E. A. Carter, Rock-salt structure lithium deuteride formation in liquid lithium with high-concentrations of deuterium: a first-principles molecular dynamics study, Nuclear Fusion 56 (2016) 016020. 
[26] T. Abrams, Erosion and re-deposition of lithium and boron coatings under high-flux plasma bombardment, Ph.D. thesis, Princeton University (2014).

[27] J. E. Menard, et al, Overview of the physics and engineering design of NSTX upgrade, Nucl. Fusion 52 (2012) 083015.

[28] H. W. Kugel, et al., NSTX plasma operation with a liquid lithium divertor, Fusion Engineering and Design 87 (2012) 1724-1731.

[29] M. Jaworski, et al., Liquid lithium divertor characteristics and plasma-material interactions in NSTX high-performance plasmas, Nucl. Fusion 53 (2013) 083032.

[30] N. Itou, et al., Rapid diffusion of lithium into bulk graphite in lithium conditioning, Journal of Nuclear Materials 290-293 (2001) 281-285.

[31] A. Brooks, M. Jaworski, K. Tresemer, Thermal and structural performance evaluation of the NSTX-U high-z divertor, Fusion Science and Technology in preparation.

[32] H. W. Kugel, et al., The effect of lithium surface coatings on plasma performance in the National Spherical Torus Experiment, Phys. Plasmas 15 (2008) 056118.

[33] V. A. Evtikhin, I. E. Lyublinski, A. V. Vertkov, N. I. Yezhov, B. I. Khripunov, S. M. Sotnikov, S. V. Mirnov, V. B. Petrov, Energy removal and MHD performance of lithium capillary-pore system for divertor target application, Fusion Engineering and Design 49-50 (2000) 195-199.

[34] I. Tazhibayeva, O. Pivovarov, G. Shapovalov, A. Azizov, Tokamak KTM progress activity for preparation on first plasma start-up, Proceedings of the $22^{\text {nd }}$ IAEA Fusion Energy Conference (2008) FTP/P6-08.

[35] A. F. Mills, Heat Transfer, 2nd Edition, Prentice Hall, Inc., Upper Saddle River, New Jersey, 1999. 
[36] M. A. Abdou, The APEX Team, A. Ying, N. Morley, S. S. K. Gulec, M. Kotschenreuther, S. Malang, S. Zinkle, T. Rognlien, P. Fogarty, B. Nelson, R. Nygren, K. McCarthy, M. Z. Youssef, N. Ghoniem, D. Sze, C. Wong, M. Sawan, H. Khater, R. Woolley, R. Mattas, R. Moir, S. Sharafat, J. Brooks, A. Hassanein, D. Petti, M. Tillack, M. Ulrickson, T. Uchimoto, On the exploration of innovative concepts for fusion chamber technology, Fusion Engineering and Design 54 (2) (2001) 181-247.

[37] A. E. Scheidegger, The physics of flow through porous media, 3rd Edition, University of Toronto Press, Great Britain, 1974. 